\title{
Panel I: Present State of Industrial Development of Devices
}

\section{JANICE JENKINS, REPORTER; ROBERT ARZBAECHER, MODERATOR; and PANELISTS; RICHARD MARTIN, EDWIN DUFFIN, PETER TARJAN, and NORMA GILLI}

From the University of Michigan, Ann Arbor, Michigan, and the Pritzker Institute of Medical Engineering, ITT Center, Chicago, Illinois

Four representatives of the pacemaker industry served as panel members for a presentation and open discussion of the present state of industrial development of devices. Each panel member made a brief presentation describing his or her own company's approach to antitachycardia pacing. The panelists had been encouraged to provide as much detail about novel features as proprietary considerations would allow, as well as device limitations and major problems. Summaries of the initial presentations appear below, followed by the discussions which ensued.

Dr. Martin: Traditionally, tachyçardias have been classified into two basic categories: those resulting from abnormal automaticity, and those produced by a rapidly circulating impulse in an anatomically or functionally determined reentrant pathway. Reentrant tachycardias are readily initiated and terminated by pacing. Termination depends upon the delivery of a pacing pulse of sufficient prematurity to "collide" with the tachycardia wavefront, thereby interrupting the circus pathway. Success in termination depends on several factors, including the type and nature of the tachycardia, the proximity of the pacing electrode to the tachycardia circuit, the reliable detection of the tachycardia, and the delivery of impulses at the proper time.

Accepted forms of pacing for tachycardias include overdrive pacing, underdrive pacing, single or double extrastimuli, train pacing, and simultaneous pacing at two (or more) sites.

The mechanism involved in tachycardia over- . drive pacing (often referred to as burst pacing) requires pacing at a rate in excess of the tachycardia

Address for reprints: Janice Jenkins, Ph.D., Department of Electrical and Computer Engineering, University of Michigan, Ann Arbor, MI 48109, U.S.A. with $1: 1$ capture for a few impulses, followed by abrupt cessation of pacing.

A specific example of an automatic burst pacemaker is the CyberTach* 60 . This pacemaker was developed approximately $5 \frac{1}{2}$ years ago and has been in clinical use for the last 4 years. Prior to the time of its introduction, some success had been achieved with patient-activated devices.

The CyberTach 60 circumvents the problems associated with patient-activated systems. The device, after automatically detecting the tachycardia, delivers a rapid burst of pulses at a programmable rate and duration. Tachycardia detection ị based upon the presence of 7 consecutive intrinsic intervals with a cycle length shorter than the programmable tachycardia criterion.

In addition to its burst capabilities, the device also operates as a conventional demand pacemaker.

To date, there have been 172 clinical implantations of the CyberTach 60; 114 atrial applications and 58 ventricular applications. Clinical evaluation of this system has been limited to a relatively small number of institutions which have the capability of performing the thorough electrophysiologic examination required prior to implantation. Through the use of intracardiac recording and programmed electrical stimulation, the mechanism of an arrhythmia is identified and localized. The tachycardia is then initiated and terminated with programmed stimulation. These initial studies are of paramount importance in screening out patients in whom burst pacing is ineffective or potentially dangerous. In atrial applications of programmed stimulation this screening process includes discovery of the presence of a concealed accessory pathway with a short effective refractory

*Intermedics, Inc., Freeport, Texas, U.S.A. 
period which can result in transmission of the rapid atrial stimulation to the ventricle. In ventricular applications, acceleration of the tachycardia or a degeneration into ventricular fibrillation obviously precludes the use of an automatic burst pulse generator. These electrophysiologic studies are often performed concurrently with antiarrhythmic drug therapy used to supplement the pacemaker therapy.

A second form of tachycardia-treating pacemaker is contained as an integral algorithm to Intermedics' COSMOS, a new DDD pacemaker. Much has been written concerning a very special form of tachycardia commonly referred to as a pacemaker-mediated tachycardia. A pacemaker-mediated tachycardia can occur with any atrial tracking dual-chamber pacemaker when $\mathrm{A}-\mathrm{V}$ dissociation is followed by $\mathrm{V}$ to $\mathrm{A}$ conduction beyond the postventricular atrial refractory period. The first order of business is to prevent the initiation of such a tachycardia by appropriate extension of the atrial refractory period. However, if the tachycardia is initiated, a simple algorithm which detects operation precisely at the upper rate limit, drops the 16 th ventricular spike and then reestablishes $\mathrm{A}-\mathrm{V}$ association is an effective form of tachycardia termination.

Dr. Duffin: Within the past few years a renewed interest in electrical control of tachycardias has spawned a host of devices with widely varying descriptions. ${ }^{1-9}$ Implantable devices with energy outputs ranging from 30 millionths of one joule to 40 joules, using bursts, premature stimuli, scanning of rate and/or pulse position, synchronous or asynchronous shocks, and with masses varying from 45 to 250 grams are available to investigational items, custom prescription devices, or fully marketed systems. Some deliver therapies automatically while others require patient or physician intervention. Yet, despite this broad menu, it is estimated that antitachycardia stimulators account for less than 1 percent of all cardiac pulse generator implants. The primary reasons for this include availability of well-entrenched alternative therapies, fear of inducing more serious arrhythmias, and technical limitations in available pulse generator designs. Technical advances will undoubtedly reduce these barriers within the next few years, yet current state-of-the-art devices are already achieving impressive results. Three representative systems are described in order to indicate the current level of achievement.

Despite the availability of automatic antitachycardia devices, limitations in the current detection algorithms have created a niche for flexible, manually activated devices. One such system currently under investigation is referred to as the Interactive Tachycardia System* which is comprised of three major components: (1) an external stimulator called a prescription formulator; (2) an implantable lithium-powered single chamber pulse generator with bidirectional telemetry; and (3) a hand-held patient transmitter. The battery-powered prescription formulator is used to develop an appropriate therapeutic stimulation sequence, connected to the patient via a conventional catheter. The stimulation sequences available include a basic drive train, 1 to 99 premature extrastimuli utilizing 3 independent fixed or rate-adaptive intervals, automatic pulse position and/or count scanning, and fixed frequency or rate-adaptive bursts. Two sequences may be stored for rapid recall, and the system can be programmed for automatic detection of tachycardias using rate and count as criteria.

When a therapeutic regimen has been determined, the prescription is placed into the memory of a microprocessor-based patient transmitter. The patient receives an implantable antibradycardia/ antitachycardia pulse generator which incorporates bidirectional telemetry. Each cardiac event sensed by the pulse generator is indicated in the telemetry signal, and the pulse generator delivers output pulses upon receipt of appropriate telemetry from the patient transmitter or the radio frequency head of the prescription formulator.

In operation, a symptomatic patient places the transmitter over his implanted pacemaker and, if telemetry confirms an appropriate cardiac rate, the prescribed synchronous stimulation sequence is generated up to the maximum number of attempts established by the prescription.

Should the need arise, the physician can use the prescription formulator noninvasively to evaluate his patient's current status by simply placing the

\footnotetext{
*Medtronic Inc., Minneapolis, Minneșota, U.S.A.
} 
$\mathrm{RF}$ head of the prescription formulator over the implanted pulse generator. The telemetry link then allows the system to function exactly as if the external stimulator were connected directly to the patient's implanted catheter.

Devices designed for electrical control of tachycardias have most heavily concentrated on techniques to terminate the tachycardias once they have begun, yet the number of episodes requiring therapy may be minimized if appropriate antibradycardia support is available. ${ }^{10-12}$ Current technology makes possible the provision of automatic antitachycardia therapies in combination with stateof-the-art antibradycardia pacing modalities.

An illustrative example, the Symbios ${ }^{\mathrm{TM}}$, is a 55 gram, ten-millimeter thick, bipolar DDD pacemaker offering the full panoply of antibradycardia characteristics including programmable modes, rates, AV delays, refractory periods, amplifier sensitivities, output amplitudes, output pulse widths, and rate-limiting behaviors. In addition, the device provides automatic tachycardia termination by means of atrial bursts and single- or dual-chamber, dual-demand pacing. Burst rates, burst counts, and tachycardia rate thresholds can all be programmed noninvasively, and a resettable monitoring flag provides a record of tachycardia detection. The system further provides for operation with an enhanced patient-interactive device similar to that associated with the Interactive Tachycardia System described earlier. Therapeutic options are thus broadened to include flexible premature extrastimulus sequences, rate and pulse position scanning, and ventricular bursts. These prescriptions are developed by the clinician using the pacemaker programmer which functions as a prescription formulator, and which also is used by the physician to place the prescription directly into the patient transmitter.

As antiarrhythmic pacemakers become increasingly flexible, they pose more severe interpretive problems for the clinician. Aware of a growing level of concern in this regard, manufacturers are attempting to ease the burden by including features which serve as user aids. An example of one such aid is the inclusion in the Symbios of a Marker Channel ${ }^{t M}$ function. The pulse generator Marker Channel telemeters signals which, when formatted on an ECG machine or programmer display, indicate to the user what the pacemaker is doing.
These signals substantially simplify ECG interpretation and provide a ready basis for creation of self-diagnostic pacemaker systems. The Symbios is currently in clinical evaluation throughout the world.

One widely utilized electrical antiarrhythmic regimen is cardioversion, the transthoracic delivery of moderate energy shocks synchronous with ventricular depolarization. Recent studies have indicated that cardioversion can be achieved with mean energy levels of approximately 0.5 joules through the use of appropriately designed transvenous catheters. ${ }^{13-15}$ Cardioversion can be simpler to prescribe than complex low-level stimulation sequences, and offers the potential for safely treating not only ventricular tachycardia, but also atrial fibrillation. The energy levels required for transvenous cardioversion can be provided by a relatively small implantable device weighing less than 100 grams.

The Medtronic Model 7210 Implantable Cardioverter provides automatic tachycardia detection based on programmable rate, count, and acceleration criteria. Each episode of tachycardia triggers delivery of up to 5 therapeutic shocks, the first 3 attempts at programmed energy levels ranging from .06 to 2.0 joules and, should these fail, two final attempts at the maximum level of 2.0 joules. The system also includes a multiprogrammable VVI pacemaker, facilities for performing noninvasive premature stimulation studies for patient evaluation, and telemetry of program settings, electrograms, and marker channel information. The device weighs 95 grams, and is 19 $\mathrm{mm}$ thick. With appropriate system revisions it is anticipated that future editions of the cardioverter will be sufficiently efficient that defibrillation may be possible with energy levels currently being used for cardioversion.

The efficacy and safety of the implantable cardioverter concept remain to be established in human clinical trials which are now in progress.

Dr. Tarjan: The concept for Orthocor* was first proposed more than ten years ago, but due to priority conflicts, its clinical introduction was delayed

${ }^{\star}$ Cordis Corp., Miami, Florida, U.S.A. 
until 1980. The Orthocor system consists of an implant, an overdriver and a prescriber. The Orthocor Prescriber (O.P.) is a computer-based instrument in the electrophysiologist's laboratory and with it, the physician can set up stimulation protocols to be delivered directly to cardiac leads by the Orthodriver III (O-III), an external, handheld, battery-powered microcomputer. The O-III can also store in its memory a sequence from the O.P. that can be activated by the patient and its pulses conductively coupled through the implanted electrodes to the Orthocor implant, which in turn performs the stimulation and provides time references by detecting spontaneous cardiac events.

The O-III provides electrical isolation between the computer and the patient. The Orthocor implant normally functions as an inhibited (AAI or VVI) pacemaker, but when a magnet is placed near it, it becomes a synchronous (AAT or VVT) type pacer. As it cannot distinguish between cardiac events and certain man-made pulses, such as those produced by O-III, it will detect the pulses from $\mathrm{O}$-III and stimulate the heart in synchrony.

Over the past 3 years, 74 of these Orthocors have been implanted in 2 forms: 5 with asynchronous and 69 with synchronous modes of response to patient activation. Fifteen have been used with single atrial leads and 59 with ventricular leads.

The available stimulation patterns have only one limitation: the minimum interval between consecutive pulses is $170 \mathrm{~ms}$. The possibilities are wide open. It was our expectation that the Orthocor program would establish the safe and necessary range of parameters in an automatic tachycardia-terminating implant. The study is still in progress but thus far, it has raised more questions than it has answered.

Orthocor II is an automatic device approaching clinical trials. It offers all of the patterns which have been useful in the patient-activated mode and some hypothetically useful ones. New protocols are being proposed and evaluated in electrophysiology (EP) laboratories. Advances are expected with two-channel stimulation which may now be evaluated with Gemini ${ }^{*}$ pacers operating in the DDT mode. Recent experiences in this application have been encouraging, especially for

${ }^{\star}$ Cordis Corp., Miami, Florida, U.S.A. noninvasive serial drug evaluation by electrical challenges to the heart.

The capabilities of Orthocor II include not only automatic response with any one of the programmable patterns, but also automatic iterative adjustments in the parameters if the tachycardia persists. Its other features include extensive memory capabilities for keeping track of such events as the frequency of premature beats, tachycardias, etc. Its small size should make it cosmetically acceptable for younger patients.

The implantation of an antitachycardia device must be preceded by a sequence of EP studies. The instrumentation used in the laboratory must be capable of testing the effectiveness of the contemplated implant. We have recognized this need and have evolved a series of laboratory stimulators to provide the flexibility necessary for this rapidly changing clinical field of study. The O.P. was followed by the EPS I, which added automatic pacing support in case of bradycardia. The EPS II is an advanced two-channel version with even broader capabilities. As these instruments are software-organized, the evolution of new stimulation modes will not require replacement or hardware modification of the instruments. The most interesting feature of these stimulators is their ability to store for future use 4 protocols which can be activated by two keystrokes. This permits the clinician to set up in advance various termination sequences to be called up the moment the tachycardia is electrically induced. This saves time and renders the studies safer.

Ms. Gilli: For over 100 years electrical stimulation has been used to terminate paroxysmal tachycardia. ${ }^{16}$ Modern implantable pacing methods include underdrive, overdrive, and the extrastimulus method. ${ }^{17,18}$ Underdrive pacing is asynchronous stimulation at a rate less than that of the tachycardia. This may be regarded as an unrefined extrastimulus method since the stimulus will eventually "scan" through the tachycardia cycle. ${ }^{19}$ This method is considered safe but has limited application owing to the critical dependence on the site of stimulation. Overdrive pacing consists of pacing at a rate faster than the tachycardia. This method is successful in terminating tachycardias but may produce unwanted arrhythmias ${ }^{19}$ Burst pacing in the atrium has proven a very effective technique for termination of supraventricular tachycardia 
(SVT) but the stimulation is randomly delivered and may cause atrial fibrillation. ${ }^{20}$ The delivery of critically timed extrastimuli has been shown to terminate the majority of SVT but success may be critically dependent on lead placement and timing.

Critically timed extrastimuli have been effective in interrupting a reentry pathway. ${ }^{21}$ It has been found in some patients that two or more stimuli may be required to terminate high rates of tachycardia. Overdrive pacing has been one of the most effective means of terminating tachycardia. In the past, standard burst pacemaker limitations included the following: if the rate was too slow, the pacemaker would drive the tachycardia, while rates that were too high might precipitate dangerous arrhythmias. In addition, prolonged repetitive bursts could cause hemodynamic deterioration. If the tachycardia were not terminated by the burst, each subsequent recognition would cause the pacemaker to continue to repeat the same ineffective sequence. ${ }^{22}$

The synchronized scanning burst pacemaker overcomes these limitations. In 1981, Telectronics introduced the Programmable Automatic Arrhythmia Reversion (PASAR Model 4151) pulse generator for clinical studies. The PASAR can be programmed to deliver a single or coupled extrastimuli. The interval from the cardiac signal (confirming tachycardia) to the first extrastimulus (initial delay) can be scanned through a 90-ms window, in 156 ms steps from the programmed value. The interval between the 2 extrastimuli (coupled delay) is programmable and remains constant throughout the scanning of the initial delay. To date, there have been over a 100 units implanted in 38 centers around the world.

Features in the new PASAR (Model 4171) include tachycardia detection, in which four consecutive cycles must occur at a rate faster than the programmed tachycardia detection rate for tachycardia confirmation. The tachycardia rates recognized are: $110,120,130,140,150,170$ and 200 bpm. In addition, a tachycardia rate of $65 \mathrm{bpm}$ can be specified in order to provide a method of noninvasive testing of the correct operation of the pulse generator. This facility may be used to initiate tachycardia recognition artificially and then it can be reprogrammed to test the efficacy of chosen reversion parameters. Programming burst status "FIXED" provides a synchronized "shifting" burst in which the initial delay decrements in 15 6-ms steps. Programming burst status "SCAN" results in the delivery of a synchronized burst of up to 7 pulses. Both the initial and the coupled delays decrement in 15 6-ms steps until tachycardia termination is successful. The subsequent bursts are delivered with shorter coupled delays, hence the term "CONCERTINA" burst.

\section{Problems and Future Work}

Dr. Martin: Looking to the future of antitachycardia devices, there are at least three major areas that require attention. These include suppression, detection and termination. Tachycardia suppression by pacemakers includes continuous pacing from single or multiple sites to suppress the reentrant mechanism. Although not always possible, tachycardia suppression is by far the preferred method for dealing with the arrhythmia. Very advanced devices with the capability for true "physiological"pacing will provide improvements in tachycardia suppression.

Tachycardia detection by pacemakers has been limited almost exclusively to detection of intrinsic rate. Because of the complex nature of tachycardias and exercise-induced rapid heart rates, monitoring heart rate alone can be misleading in tachycardia detection. Monitoring the derivative (rateof-change) of heart rate, as well as sensing from multiple sites, provides additional information. This information enables more reliable discrimination of reentrant or nonphysiologic tachycardias from rapid sinus rhythms.

Termination of tachycardias requires precisely delivered impulses from the pacemaker; otherwise, the pulses are ineffective or even deleterious. To be most effective, the pacemaker must be capable of executing complex pacing algorithms that are formulated from electrophysiological research. As this research progresses, newer algorithms will evolve. Total flexibility in programming, much like that available in laboratory stimulators, will be required of the pacemaker.

Tachycardia cycle lengths are dynamic; that is, they change. Therefore, in order for a device to be chronically effective, it must adapt to the changing rate which will allow the pulses to enter the termination zone reliably. I believe such adaptive mechanisms are essential. 
In addition to, or as an integral part of, adaptive capabilities is the necessity for back-up forms of therapy. If the primary mode of therapy fails to terminate the arrhythmia after a programmed number of attempts, a secondary modality is initiated. The successful parameters are stored in memory and initiated upon the next tachycardia event. Both adaptive and primary/secondary therapy are important when considering candidates exhibiting multiple foci and/or morphology and who are not, as a result, appropriate surgical candidates.

The collection and storage of diagnostic data are important functions of future devices. Examples of data include number of tachycardia events, number of termination attempts, and successful termination parameters. Storage of selected electrograms is also deemed important.

In order to achieve widescale utilization, noninvasive electrophysiological studies must be performed via programmed stimulation, using the programmer for the purpose of selecting the most appropriate parameters and for long-term followup.

Finally, although aggressive pacing techniques in the ventricular application can terminate the vast majority of tachycardias, defibrillation backup is essential.

Dr. Duffin: Much remains to be accomplished before electrical control of tachyarrhythmias becomes a widely accepted therapeutic option. The arrhythmia detection and classification algorithms are currently quite primitive, limiting the application of automatic devices to situations in which there is sharp delineation between sinus and pathologic tachycardias. This will change. Provision of back-up defibrillation capabilities in automatic antitachycardia systems will reduce concerns about inducing lethal accelerations. Much work remains, however, to achieve this without creating devices which are simply too large for widespread acceptance. The technology to permit this is within sight and, beyond offering size reductions, it could make possible cardioversion and defibrillation at substantially lower energies than are currently being used. This will afford yet another significant advance by substantially reducing the patient trauma associated with high-energy stimulation.
Secondary benefits of implantable arrhythmia control devices can be derived by including facilities for noninvasive provocative testing and monitoring/recording. These will aid the physician in following his patients' progress and prescribing necessary modifications to a therapeutic regimen. Some of today's devices provide substantial provocative test facilities, but offer only limited monitoring. This will be dramatically improved as microprocessor and sensor technology rapidly infiltrate implantable device designs.

Much work remains in establishing appropriate clinical indications for the use of electrical antitachycardia therapies. It is anticipated that many fruitful collaborative efforts between device manufacturers and the medical community will address this issue in the coming years. As these systems evolve, it is expected that they will become sufficiently flexible to be considered antiarrhythmic rather than purely antitachycardic.

In conclusion, a projected device of the near future might well be a dual chamber system with sophisticated arrhythmia detection and classification capabilities, therapeutic bursts for atrial arrhythmias, low-energy cardioversion for ventricular tachycardias and atrial fibrillation, back-up defibrillation capabilities, and provisions for noninvasive provocative patient studies and comprehensive monitoring. This is all potentially achievable within the next few years.

Dr. Tarjan: Our chairman for this panel requested that we identify those areas of difficulties which await solutions from the engineers' viewpoints. In my opinion the rapid and reliable detection of tachycardias and their classification is the most challenging problem, but this will be solved as more powerful microprocessors become available for implantable devices.

The problem that concerns me most from the viewpoint of feasibility is a small and superbly reliable antiarrhythmic device that includes an automatic defibrillator for those situations in which all else fails. It is my hope that cooperation between electrophysiologists and engineers will result in preventive means combining monitoring devices with stimulators and drug infusers. Preventive schemes might someday reduce the probability of fatal ventricular fibrillation to an insignificant level. 
Ms. Gilli: One major problem in an automatic pacing system is the ability to sense sinus rhythm owing to exercise which may exceed the tachycardia detection rate, thereby initiating the antitachycardia function. Multiple rates of tachycardia are often a problem that is very difficult to overcome. Patients may have two or more tachycardia rates, one which may be lower than the tachycardia detection rate. The inability to sense $P$-waves may result in a lack of confirmation of tachycardia. P-waves may appear adequate during normal sinus rhythm but P-wave changes during the tachycardia may not be sensed. There is at present no reliable way to monitor antitachycardia pacemakers in order to prove their safety and efficacy.

\section{Panel Discussion}

Dr, Arzbaecher: We have all talked about the problem of detection. Everyone agrees, both manufacturer and user, that the problem with detection of tachycardia is serious, yet no one has yet presented data on failures of detection. Could you give us any idea of how many times a Cybertach 60 has misdiagnosed a tachycardia, or can you lead us to any data about this question?

Dr. Martin: The device has programmable șensitivity and can be made, relatively speaking, to be very sensitive. The problem has not been in the literal detection of the tachycardia but in the detection of other things that could fool it. Because it is a bipolar system, it is to some extent free of many of the typical forms of interference associated with pacemaker therapy (particularly unipolar pacemaker therapy), like myopotential inhibition. Generally speaking; however, there have been no problems associated with the detection of the tachycardia. Although I am sure that it happens on occasion, I am not aware of the actual incidence level.

Dr. Arzbaecher: Do you have a mechanism for other than anecdotal recording of remarks by patients who feel symptomatic and don't get treated, or who feel they have been treated but don't want it? Besides that, does Intermedics have any mechanism in its clinical studies for acquiring, via Holter or other techniques, specifics about the activity of the Cybertach 60?

Dr. Martin: Well, certainly we have done a lot of Holter monitoring and we have documented the appropriate performance of the device under normal day-to-day activity with the patient. We do have a flag which will indicate that, in fact, a tachycardia has been detected, that the tachycardia-treating element of the pacemaker has been programmed on, and that a tachycardia treatment has been delivered. However, it only indicates that it has done it at least one time; it's not that sophisticated and has no ability to count.

Dr. Arzbaecher: Does anybody in the audience have comments on this particular point?

Member of audience: Occasionally it is desirable to pace in the coronary sinus for a variety of reasons but there are some theoretical problems associated with sensing in the coronary sinus in these devices. My specific concern is the problem of sensing an atrial and a ventricular deflection and having the pacemaker "think" that a tachycardia is present during sinus rhythm.

Dr. Paul Gillette: We use the Cybertach 60 often in testing in the cath lab and we have seen oversensing if we use the coronary sinus lead fairly frequently because the atrial and ventricular electrograms are very similar in size in the coronary sinus. I think the right atrial appendage or some other place is by far the more appropriate place to put the electrode. As a response to the earlier discussion, we have 7 implants in relatively young patients and fortunately 6 of them have rapid tachycardias so we can use the higher setting to detect the tachycardia. To the best of my knowledge, none of those has trouble with the sinus tachycardia problem even though we have done exercise tests on all of them. In the seventh one, we must use the lower setting, the 137 , criterion to sense his tachycardia which has a rate of about 150 . On the treadmill he will oversense reproducibly, or sense tachycardia and then burst into it. He likes to be very active and has learned to feel his pulse, when he jogs, and slow down a little bit when it gets up to 137 . 
Dr. Arzbaecher: What do you think is the prospect for a very simple derivative of rate sensor, such as a delta, as Dr. Zipes called it today, in which you measure the acceleration, not just the event?

Dr. Gillette: That will work in a large majority of patients. Rate criteria plus a delta, I think, will solve over $90 \%$ of the problems.

Dr. Seymour Furman: I would like to comment about the rate delta, and about coronary sinus leads. Regarding rate delta, there are a series of algorithms which should take certain functions into account so that one can design a computer program which will distinguish between various kinds of tachyarrhythmia, and sinus tachycardia that is absolutely normal, from a whole host of other things. Going back to coronary sinus leads, I think it is absolutely essential to distinguish between unipolar and bipolar leads. Paul, were yours unipolar or bipolar in the coronary sinus?

\section{Dr. Gillette: Bipolar.}

Dr. Furman: Our experience is quite different from yours. We have invariably been able to find the position in the coronary sinus in which the intrinsic deflection in the electrogram of the atrial depolarization is of significantly higher frequency and of significantly greater sharpness and clarity than the far-field deflection which the ventricle will be at that position. So when we have used coronary sinus pacing - and we have-in the bipolar mode, we have been able to avoid sensing the ventricular contraction. We have not used it for antitachycardia pacing. We have not done so because antitachycardia modes became available at almost the same time as did good J electrodes for the right atrial appendage. We felt that given the options, we were wiser to stay in the right atrial appendage which is geographically further than the ventricle. However, when a well-designed bipolar lead is placed properly in the coronary sinus, it can detect a P-wave, which will be a far better signal of the atrial depolarization than the far-field will be, and it rejects the ventricular electrogram substantially or almost completely.

Dr. Jerry Griffin: I'd like to comment on both points-first on the coronary sinus. I think that there is a very real problem and our experience has been similar to Dr. Gillette's and somewhat in contrast with Dr. Furman's in terms of antitachycardia pacing. It is quite acceptable for general atrial pacing because you also have the refractory period to help out, but you must remember that with automatic antitachycardia devices, the refractory periods are of necessity very short and they do not cover up the ventricle at all. If you want to exercise the use of programmable sensitivity you may find yourself limited by a ventricular electrogram inserting itself into that. I actually feel fairly strongly that the coronary sinus should be avoided if you're doing automatic antitachycardia pacing because the selectivity is just much better further away, even for bipolar leads. As for the second question, we have had experience with about 11 patients now with automatic tachycardia devices. At the present time, of course, the only documentation we get is by ambulatory monitoring. Out of that group we've only had one adult patient who had clinical problems with pacing in sinus tachycardia and those were actually fairly easily solved. Just by rate criteria alone, we haven't had a major clinical problem in adult patients.

Dr. John Camm: I'd like to ask the panelists about another aspect of tachycardia recognition. In particular, I am referring to when a pacing intervention has been made and it is necessary to reconfirm that tachycardia is present or to decide whether sinus rhythm has been reestablished. This particular point is very important in pacemakers which rely on memory, for example, and more so in pacemakers which actually employ learning techniques, as the new Siemen's P33 system does. As far as I'm aware, nobody has addressed this specific problem of reconfirming tachycardia or establishing that sinus rhythm is present after a pacing sequence or within a continuing pacing burst.

Dr. Duffin: I think in most cases the method of the automatic system is one of looking to see if the escape mechanism following the burst or the scanning process still qualifies for the tachycardia condition. If it does, the system should generally try again, and if it doesn't, they will ignore it and assume that the tachycardia was broken. In the systems that we have worked with so far, that has 
rarely been raised as a difficulty. We are not aware of very many instances.

Dr. Camm: I think I have seen two situations in which it might well have been a problem. I think that Karel den Dulk showed a slide in which one tachycardia was broken to reveal sinus rhythm with a cycle length of $400 \mathrm{~ms}$. That was one possibility, although obviously it wasn't a problem in his particular case, but it could have been a problem. I think also that Doug Zipes showed a slide in which atrial fibrillation was provoked by the implantable cardioverter. It was true that ventricular tachycardia in that case did continue and therefore the cardioverter was quite justified to attempt termination again. But it might not have done and the atrial fibrillation might have deceived the cardioverter into "thinking" that the tachycardia for which it was primarily active was still present.

Dr. Duffin: Yes, I think one of the weaknesses seen in some of the attempts to detect tachycardia stems from the efforts to use onset characteristics. I think that any sequence that depends on onset will ultimately give difficulty or result in a residue of situations in which it won't work. I think that the truly successful tachycardia-discrimination process will have to be one which will come in the middle of an ongoing tachycardia and clearly define what it is without "seeing" how it started. It will have to deal with the problem of sorting atrial and ventricular rhythms. The major problem has been with patients whom you would like to treat with an automatic ventricular system, but atrial rhythms confuse the system. When you look at the incidence of how many times a system does not work properly, then we have been fortunate in not seeing too many of those. This is because the users have screened their patients very carefully. I know of a number of lost potential implants, and appropriately lost, because the current algorithms wouldn't be adequate for sorting out their tachyarrhythmias from other normal rhythms.

Dr. Tarjan: I can't speak for the whole industry, but it seems the ideal situation would be to store segments of the electrocardiogram for later inspection, and that requires a sizable memory space in the implant. I don't think you'll see this in the immediate future except for some minimal func- tions stored for later retrieval and some superficial analysis. What is possible, of course, is to count the number of times the antitachycardia device is activated; the duration of it, and perhaps some other characteristics, such as the specific rates seen. These things can be stored without overburdening the power supply and the capability of the electronics. On the other hand, there are large-size memories on the horizon and in 5 or 6 years, perhaps, it will be feasible to store segments of a few dozen episodes of tachycardia.

Dr. Arzbaecher: Does anyone have any opinions about the way a clinical study should be done, a way that measures safety and efficacy on a 24hour-a-day basis? What things would you like to have in order to track your patient to that extent?

Dr. Michael Bilitch: One possibility is what we've been discussing, namely, the question of storing more on the device. Another possibility is to see some significant improvements̃ in long-term monitoring. We're basically saddled with $24-$ or 48 hour Holter monitoring. Industry should be able to develop something small enough to store inside the pulse generator, such as an improved external reporting system that will do the processing on the patient rather than on another device 50 miles away. I think that if that's ever available, it would be mandatory for industry and the clinical investigators to use that capability for clinical trials.

Dr. Martin: That is my opinion, also. However, I believe that before we get to the point where we are actually doing the kind of storage within the pacemaker as suggested, we probably will be doing more of it externally with fairly sophisticated systems of the kind that are now beginning to come into general use.

Dr. Nanette Wenger: Do you have any information as to how close the recognition algorithms used by ambulatory recorders are to the recognition algorithms used by pacemakers? Some of the difficulty we have had in the reports that we may be getting off the recordings may relate not to the fact that it's a different arrhythmia, but that it's a different pattern for recognition. 
Dr. Martin: I'm personally not aware that the recognition algorithms are the same at all. What we do in terms of recognizing a ventricular event in the pacemaker is relatively primitive in technical terms, but it does appear to work fairly reliably. Remember, all we're interested in knowing is whether there is an event occurring. We do some elementary forms of filtering the $\mathrm{P}$ waves and $\mathrm{T}$ waves, and we have refractory periods to help us along, but that's fairly elementary from the point of view of actual recognition of specific events.

Dr. Arzbaecher: Do you refer to the computer-based arrhythmia monitors that are in coronary care applications or in Holter scanning applications?

Dr. Wenger: Holter scanning applications. They probably have more problems detecting supraventricular tachyarrhythmias than others, but some of the difficulty in terms of the reporting may relate to the fact that if the accuracy of function is done with an ambulatory recorder-based evaluation, it may not be the problem of the pacer but the problem of the recorder and its evaluation.

Dr. Stanley Briller: I would like to return to the problem of evaluation of efficacy. I don't think the manufacturers should adopt the position that it's not really germane to the present problem. I think it is very germane. You may use this device and the patient may feel well. This doesn't prove that you are doing something useful or good to that individual. These situations change from time to time. The tiny coronary artery supplying the little trigger area may thrombose off and the patient may never have an arrhythmia again for the rest of his life. There must be some proof of efficacy. On the other side of the coin, the unit may misbehave in some way or another and the patient may be one of these oblivious souls who never says anything to his physician and winds up dead in a bus station someday.

Dr. Arzbaecher: Norma, you are involved in clinical studies at Telectronics. Do you feel, too, that the clinical studies have not been properly designed for acquiring real efficacy data?

Ms. Gilli: It's very difficult. Right now the major problem I have is finding the patient in tachy- cardia while he's being monitored. I can induce him but that doesn't mean it's the same tachycardia that I'm going to break, because a lot of my patients have more than one. We have a very complex follow-up and because of it, we have trouble getting forms back. It's 12 pages long and the bottom line says, "Does this patient have any episodes he remembered, did he have any episodes not reverted?" This relies on the patient to report this data. There's one patient who comes in and says, "I'm in tachycardia now." When we hook him up to an ECG we find that he's got a rate of 80 . There are some patients who don't know, but we must ask them that question, because right now we don't have the ability to Holter, except externally.

Dr. Briller: I read Holters every day, and people write down palpitations and shortness of breath when there's nothing going on. There may be a 20-second ventricular tachycardia and they don't turn a hair. So, evaluation of symptoms from patients is very, very difficult. In fact, it's impossible.

Dr. Wenger: What we required on our original protocol was one Holter every 6 months. I ask now for one every 3 months, which is the regular routine follow-up. In the new unit, every single follow-up will be a Holter because there's no way you can bring a patient into the office and tell what's going on. It's very difficult. If you want to get the product to prove what you said, you've got to prove that the unit reverted spontaneous episodes and not just induced episodes.

Dr. Tarjan: Perhaps we heard an indirect endorsement of the patient-activated unit which would get the patient out of the malaise and we could keep track of him quite easily.

Dr. Briller: Well, that's not completely a solution either, because many patients have no symptoms at all when they have an arrhythmia. At least not right away. But it's a variable commodity and it can range from severe to nothing.

Dr. Martin: I think Dr. Briller has opened up a Pandora's box here.

Dr. Robert Hauser: I think that it must be understood that all the protocols, at least that I'm aware 
of, for the implantation of an antitachycardia device require that the patient have symptomatic tachycardia. I don't know of any patient in whom we've implanted an antitachycardia device who has not had severe symptoms that were uncontrolled by medical therapy. Therefore, I suspect if we did have a tachycardia registry, as was suggested earlier in the day, we could probably pool our data and I would suspect that we would find that most of our patients, because of the thorough testing that has been performed, have not only been relieved of their symptoms, but also many of them have not reentered the hospital because of their tachyarrhythmias. Furthermore, the kind of data that Dr. Mirowski showed earlier today, namely an improvement in actuarial survival, is the kind of information I think that all of us are interested in. I think the data are here, that they can be collected, but I agree that we have not yet seen all the information that we'd like to see to meet your requirement.

Dr. Briller: I perfectly agree, if the intention for now and for the future is to put these devices only in people who are symptomatic. However, knowing the way the world goes, I doubt that that will be a future fact. I think that this data will be extended, if this data continues to relieve symptoms and be a success actuarily.

Dr. Wenger: Do we have any information, either from the clinicians or from the companies of the patients, that symptomatic tachycardia reverted? Have any of those patients had nonsymptomatic tachycardia that the pacemaker has reverted?

Dr. Duffin: I think Fisher has reported cases where the patients who have been treated were not even aware of the episodes. There are many in the supraventricular category. With respect to Dr. Briller's question, Dr. Neuman from Bonn published a report about a very large series of patients who were treated with several different automatic burst pacemakers and showed the hospitalization records and the symptom records and the returnto-work capabilities that were restored to these patients. I think there is data building. I get a sensation here that it's us against them, and I don't think that's the way it ought to be. I think the companies are trying to work with the physicians, the medical community, to the extent that you can design better studies and help us. We certainly want to prove that our devices are worthwhile and reliable so that we can help sell them.

Dr. Furman: Ed Duffin said a little bit of what I was going to say. First of all, I'd like to say a kind thing for the physicians in the audience. We're at least as interested in demonstrating efficacy as the manufacturers are or the FDA is; that's an important consideration. I saw this conversation drifting in the direction of "What are you four up there doing?" and I protest that. I think we are all working with this thing together. Now, it would be valuable at this point to provide an infinite amount of memory. I'm not sure, and I agree with Ed Duffin again on this, that that's now available in an implantable form of reasonable size. It would be very easy to undertake daily Holter monitoring and, with that, I'd like to recount the case of one of the Cybertachs in which the company was immensely helpful when we were very much trying to determine whether this unit was effective. We arranged to put aside one of the hospital Holter monitors and assigned it to a patient day in and day out. Well, after four months there had not been an episode, and we gave up. Six weeks later, there was an episode of something and the patient died and we didn't catch it. Now, it seems to me that under those circumstances it is easier to ask the question, "When do you give up?" I think we gave it a real shot, but when do you give up? I think this is exactly the time when a national tachycardia registry in which the input of a greater pool of income or money or resources than is available either to the manufacturers or to an individual physician should be brought to bear. I can't imagine where a greater source of funds would be available, but if there is such a source, perhaps they may be listening at this moment.

Tibor Nappholz from Telectronics: I just wanted to comment, related to follow-up, on the PASAR. We are very wise to the fact that follow-up is such an incredibly difficult thing and we are wise to it after the event. The way we visualize it is that the telemetry itself will be capable of a link to an external monitoring system and the actual microprocessor system in the pacemaker will be capable 
of a duality of operations. It will either be able to store information into the pacemaker or transmit it out to the Holter monitor. So there is a desperate need for that system actually to generate diagnostics and it is important that these diagnostics are available for continuous follow-up. There is no way that a complex system can actually be released to interact with another complex system like a patient and not have appropriate follow-up. I believe that it is imperative, in fact, that this be

\section{References}

1. Fisher, J., Kim, S., Furman, S., et al.: Role of implantable pacemakers in control of recurrent ventricular tachycardia. Am. J. Cardiol., 49:194, 1982.

2. Medina-Ravell, V., Maduro-Maytin, C., PortilloAcosta, B., et al.: Follow-up of twenty-five patients with implantable antitachycardia DVI, MN pulse generators. Presented at the VIIth World Symposium on Cardiac Pacing, Vienna, 1983.

3. Mirowski, M., Mower, M., Reid, P., et al.: The automatic implantable defibrillator. PACE, 5:384, 1982.

4. Griffin, J., Mason, J., and Calfee, R.: Clinical use of an implantable automatic tachycardia-terminating pacemaker. Am. Heart J., 100:1093, 1980.

5. Benditt, D., Kriett, J., Tobler, H., et al.: Cardioversion of atrial tachyarrhythmias by low-energy transvenous technique. Presented at the VIIth World Symposium on Cardiac Pacing, Vienna, 1983.

6. Waxman, M., Wald, R., Bonet, J., et al.: Self-conversion of supraventricular tachycardia by rapid atrial pacing. PACE, 1:35, 1978.

7. Spurrell, R.: Treatment of reentrant tachycardia using a fully implantable automatic scanning pacemaker. Int. J. Cardiol., 2:413, 1983.

8. O'Keefe, D., Curry, P., and Sowton, E.: Treatment of paroxysmal nodal tachycardia by dual demand pacemaker in the coronary sinus. Br. Heart J., 45:105, 1981.

9. Vohra, J., Hamer, A., Mond, H., et al.: Patient initiated implantable pacemakers for paroxysmal supraventricular tachycardia. Aust. N. Z. J. Med., 11:27, 1981.

10. LeClercq, J., Rosengarten, M., Delcourt, Ph., et al.: Prevention of intra-atrial reentry by chronic atrial pacing. PACE, 3:162, 1980.

11. Iesaka, Y., Pinakatt, T., Gosselin, A., et al.: Bradycardia dependent ventricular tachycardia facilitated by myopotential inhibition of a VVI pacemaker. PACE, 5:23, 1982.

12. Gould, L., Gopalaswamy, C., Chandy, F., et al.: One monitored case of sudden death. PACE, 6:238, 1983. addressed with a great deal of urgency. The immediate possibility is to use an external Holter which is going to tell you exactly what the patient is doing, how the pacemaker responds, and in fact, what the pacemaker is doing continuously. Thank you.

Dr. Arzbaecher: I thank the panel members for their presentations. Further questions can be asked this evening in the Meet the Speakers' sessions.

13. Jackman, W., and Zipes, D.: Low-energy synchronous cardioversion of ventricular tachycardia using a catheter electrode in a canine model of subacute myocardial infarction. Circulation, 66:187, 1982.

14. Zipes, D., Jackman, W., Heger, J., et al.: Clinical transvenous cardioversion of recurrent life threatening ventricular tachyarrhythmias: Low energy synchronized cardioversion of ventricular tachycardia and termination of ventricular fibrillation in patients using a catheter electrode. Am. Heart J., 103:789, 1982.

15. Fisher, J., Matos, J., and Kim, S.: The sparkling joules of internal cardiac stimulation: Cardioversion, defibrillation, and ablation. Am. Heart J., 104:177, 1982.

16. Wellens, H., Bär, F., and Gorgels, A.P.: Electrical management of arrhythmias with emphasis on the tachycardia. Am. J. Cardiol., 41:1025, 1978.

17. Spurrell, R., Nathan, A., and Bexton, R.: Implantable automatic scanning pacemaker for termination of supraventricular tachycardia. Am. J. Cardiol., 49:753, 1982.

18. Nathan, A., Camm, A., and Bexton, R.: Experience with a fully implantable, programmable, scanning, extrastimulus pacemaker for tachycardia termination. Clin. Cardiol., 5:22, 1982.

19. Camm, A., and Ward, D.: Pacing for Tachycardia Control. London, Telectronics, Buther and Tanner, 1983, pp. 30, 32-33.

20. Hartzler, G.O., Holmes, D.R., Jr., and Asborn, M.J.: Patient-activated transvenous cardiac stimulation for the treatment of supraventricular and ventricular tachycardia. Am. J. Cardiol., 47:903, 1981.

21. Wise, J., Jr.: Patient activated atrial pacing in the treatment of recurrent supraventricular tachycardia. Chest, 65:214, 1974.

22. Nathan, A., Hellestrand, K., and Bexton, R.: Clinical evaluation of an adaptive tachycardia intervention pacemaker with automatic cycle length adjustment. PACE, 5:303, 1982. 
Copyright of Pacing \& Clinical Electrophysiology is the property of Blackwell Publishing Limited and its content may not be copied or emailed to multiple sites or posted to a listserv without the copyright holder's express written permission. However, users may print, download, or email articles for individual use. 